\title{
Paradoxical Facilitation of Object Recognition Memory after Infusion of Scopolamine into Perirhinal Cortex: Implications for Cholinergic System Function
}

\author{
Boyer D. Winters, Lisa M. Saksida, and Timothy J. Bussey \\ Department of Experimental Psychology, University of Cambridge, Cambridge CB2 3EB, United Kingdom
}

\begin{abstract}
The cholinergic system has long been implicated in learning and memory, yet its specific function remains unclear. In the present study, we investigated the role of cortical acetylcholine in a rodent model of declarative memory by infusing the cholinergic muscarinic receptor antagonist scopolamine into the rat perirhinal cortex during different stages (encoding, storage/consolidation, and retrieval) of the spontaneous object recognition task. Presample infusions of scopolamine significantly impaired object recognition compared with performance of the same group of rats on saline trials; this result is consistent with previous reports supporting a role for perirhinal acetylcholine in object information acquisition. Scopolamine infusions directly before the retrieval stage had no discernible effect on object recognition. However, postsample infusions of scopolamine with sample-to-infusion delays of up to $20 \mathrm{~h}$ significantly facilitated performance relative to postsample saline infusion trials. Additional analysis suggested that the infusion episode could cause retroactive or proactive interference with the sample object trace and that scopolamine blocked the acquisition of this interfering information, thereby facilitating recognition memory. This is, to our knowledge, the first example of improved recognition memory after administration of scopolamine. The overall pattern of results is inconsistent with a direct role for cortical acetylcholine in declarative memory consolidation or retrieval. Rather, the cholinergic input to the perirhinal cortex may facilitate acquisition by enhancing the cortical processing of incoming stimulus information.
\end{abstract}

Key words: acquisition; consolidation; declarative memory; interference; muscarinic; rat

\section{Introduction}

The neurotransmitter acetylcholine (ACh) has long been implicated in cognitive functions (Everitt and Robbins, 1997; Sarter and Bruno, 1997; Bartus, 2000), but the specific contribution of cortical ACh to learning and memory remains unclear. The nature of the mnemonic effects produced by certain cholinergic manipulations suggests a role in enhancing cortical information processing to facilitate acquisition of new material (Chiba et al., 1995; Bucci et al., 1998; Furey et al., 2000; McGaughy et al., 2005), a function for cortical ACh that would be consistent with its broader role in attention (Sarter et al., 2003). Conversely, results from studies with postacquisition manipulations have suggested that ACh may be directly involved in memory consolidation. These findings, however, have been mixed, with some suggesting that ACh is necessary for consolidation of memory for tasks such as fear conditioning and inhibitory avoidance (Power et al., 2003) and others pointing toward a facilitative role for reduced levels of

\footnotetext{
Received June 1, 2006; revised Aug. 1, 2006; accepted Aug. 10, 2006.

This work was supported by a Biotechnology and Biological Sciences Research Council Research Grant to B.D.W. L.M.S., and T.J.B. and a Wellcome Trust Project Grant to L.M.S. and T.J.B. This research was conducted under the auspices of the Cambridge Medical Research Council Centre for Behavioural and Clinical Neuroscience. We thank Lizzy Blakeway and Vicki Armitage for assistance with data collection for experiment 1 and Nasr Faroogi and Amy Hufton for assistance with data collection for experiment 2.

Correspondence should be addressed to Dr. Boyer D. Winters, Department of Experimental Psychology, University of Cambridge, Downing Street, Cambridge CB2 3EB, UK. E-mail: bdw23@cam.ac.uk.

DOI:10.1523/JNEUROSCI.2319-06.2006

Copyright $\odot 2006$ Society for Neuroscience $\quad$ 0270-6474/06/269520-10\$15.00/0
}

cortical ACh in declarative memory consolidation (Hasselmo, 1999; Gais and Born, 2004a,b; Hasselmo and McGaughy, 2004).

The correlation between cholinergic system dysfunction and cognitive impairment in Alzheimer's disease (AD) has been very influential in the implication of ACh in learning and memory functions (Perry et al., 1978; Bartus et al., 1985). The most prominent early cognitive symptom in $\mathrm{AD}$ is a disruption in the ability to acquire new declarative memories (Morris and Kopelman, 1986). Declarative memory has been studied in animals to a large extent using tests of object recognition memory; indeed, it has been suggested that object recognition tasks provide a relatively pure assessment of declarative memory function (Manns et al., 2000).

A structure known to be crucial for object recognition is the perirhinal cortex (PRh) (Zola-Morgan et al., 1989; Meunier et al., 1993; Mumby and Pinel, 1994; Buffalo et al., 1998; Winters et al., 2004), and recent studies have implicated the cholinergic input to the PRh in this form of memory (Tang et al., 1997; Warburton et al., 2003; Abe et al., 2004; Turchi et al., 2005; Winters and Bussey, 2005b). The present study, therefore, examined systematically the involvement of PRh ACh in the different stages of the spontaneous object recognition task (Ennaceur and Delacour, 1988). Specifically, we tested the effects of transient intra-PRh blockade of muscarinic cholinergic receptors with scopolamine during sample presentation (encoding/acquisition), during the choice phase (retrieval), and during the retention interval (storage/con- 
solidation). We have previously demonstrated involvement of PRh in these three stages of object recognition memory, and the pattern of these past results has suggested that the object trace is consolidated within the PRh by 40 min after the sample presentation (Winters and Bussey, 2005a,c). In the present study, presample infusions of scopolamine disrupted long-term object recognition memory, whereas infusions administered during the retention interval produced a surprising and robust memory facilitation. These results suggest a direct role for cortical ACh in the acquisition, but not consolidation or retrieval, of declarative memory.

\section{Materials and Methods}

\section{Subjects}

The subjects were 108 adult male Lister hooded rats (Harlan Olac, Bicester, UK), weighing 270-320 g before surgery and housed in pairs in a room with a $12 \mathrm{~h}$ light/dark cycle (lights on at 7:00 P.M.). Different batches of rats were used for each experiment. The number of rats used in each experiment was as follows: experiment 1, 27 rats; experiment 2, 27 rats; experiment 3, 32 rats; experiment 4,12 rats; experiment 5, 10 rats. All behavioral testing was conducted during the dark phase of the cycle. During testing, rats were fed $\sim 15 \mathrm{~g}$ of laboratory chow after daily behavioral sessions to maintain weights at $85-90 \%$ of free-feeding body weight. Water was available ad libitum throughout the experiment. All experimentation was conducted in accordance with the UK Animals (Scientific Procedures) Act, 1986.

\section{Surgery}

For each experiment, all rats were implanted bilaterally with 22-gauge indwelling guide cannulas according to the following procedure. Before surgery, all animals were deeply anesthetized by intraperitoneal injection $(60 \mathrm{mg} / \mathrm{kg}$ ) of sodium pentobarbital (Sagatal; Rhône Mérieux, Essex, UK) and placed in a stereotaxic frame (David Kopf Instruments, Tujunga, CA) with the incisor bar set at $-3.2 \mathrm{~mm}$. The scalp was cut and retracted to expose the skull. Holes were drilled and the guide cannulas implanted according to the following coordinates, measured relative to the skull at bregma (Paxinos and Watson, 1997): anteroposterior, -5.5 $\mathrm{mm}$; lateral, $\pm 6.6 \mathrm{~mm}$; dorsoventral, $-6.5 \mathrm{~mm}$. The ends of the guide cannulas were, thus, located just dorsal to the main target area. The cannulas were secured to the skull using four jeweler screws and dental acrylic. Obdurators cut to extend $1.1 \mathrm{~mm}$ beyond the tip of the guide cannulas and with an outer diameter of $0.36 \mathrm{~mm}$ were inserted into the guides and remained there except during infusions. At the completion of each surgery, the skin was sutured, and an antibiotic powder (Acramide; Dales Pharmaceuticals, Skipton, UK) was applied. Animals were allowed to recover for at least $7 \mathrm{~d}$ before the beginning of behavioral testing.

\section{Histology}

After behavioral testing, rats were anesthetized by intraperitoneal injection of $2 \mathrm{ml}$ of Dolethal (Rhône Mérieux) and perfused transcardially with $100 \mathrm{ml}$ of PBS, pH 7.4, followed by $250 \mathrm{ml}$ of $4 \%$ paraformaldehyde (PFA), pH 7.4. The brains were removed, postfixed in $4 \%$ PFA at $4^{\circ} \mathrm{C}$ for $24 \mathrm{~h}$ and then immersed in $25 \%$ sucrose in PBS until they sank. Coronal sections $(60 \mu \mathrm{m})$ were cut on a freezing microtome through the extent of $\mathrm{PRh}$, and every fifth section was mounted on a gelatin-coated glass slide and stained with cresyl violet. Slides were examined under a light microscope to verify the cannula placements.

\section{Infusion procedure}

For all experiments, rats received bilateral infusions of either physiological saline $(0.9 \%$ sodium chloride, $\mathrm{pH} 7.0$; Aquapharm; Animalcare, York, UK) or scopolamine hydrobromide trihydrate $(10 \mathrm{mg} / \mathrm{ml}$ in physiological saline; Sigma, Poole, UK) on a given trial. For all conditions, the basic procedure was as follows. All infusions took place in a preparation room separate from the behavioral testing area. Animals were gently restrained by the experimenter throughout the infusion process. The obdurators were removed, and the 28-gauge infusion cannulas, which were cut to extend $1 \mathrm{~mm}$ beyond the tip of the guides, were inserted into the guides. Bilateral infusions were conducted simultaneously using two
$1 \mu$ l Hamilton syringes, which were connected to the infusion cannulas by propylene tubing. The syringes were driven by a Harvard Apparatus (Kent, UK) precision syringe pump, which delivered $1 \mu$ l to each hemisphere over $2 \mathrm{~min}$. The infusion cannulas were left in place for an addition $1.5 \mathrm{~min}$ to allow for diffusion of the infusate. The infusion cannulas were then removed, and the obdurators replaced before the next phase of the procedure (for specific experiment parameters, see below, Spontaneous object recognition). For all experiments, in each of the two habituation sessions before the beginning of behavioral testing, rats experienced a "mock" infusion identical in all aspects to the procedure described above except that the injection cannulas contained no liquid. This was done to habituate the animals to the general protocol of the infusion procedure, including insertion of the infusion cannulas and the sound of the pump.

\section{Spontaneous object recognition}

Spontaneous object recognition was conducted in a Y-shaped apparatus, as described previously (Winters et al., 2004; Forwood et al., 2005). Briefly, the Y-shaped apparatus had high, homogeneous white walls constructed from Foamalux (Brett Martin, Lancashire, UK) to prevent the rat from looking out into the room and, thereby, maximize attention to the object stimuli. The apparatus was raised $30 \mathrm{~cm}$ from the floor with walls $40 \mathrm{~cm}$ high. Each arm was $27 \mathrm{~cm}$ in length and $10 \mathrm{~cm}$ wide. The start arm contained a guillotine door $18 \mathrm{~cm}$ from the rear of the arm. This provided a start box area within which the rat could be confined at the start of the sample and choice phases of a given trial. The floor and walls of the apparatus were wiped down with a dry paper towel between rats, but otherwise were not cleaned during the experiment. A video camera was mounted above the apparatus to record all trials. Triplicate copies of the objects were obtained, which were made of glass, plastic, or metal. For any given trial, the objects in a pair were composed of the same material so that they could not readily be distinguished by olfactory cues. The height of the objects ranged from 5 to $20 \mathrm{~cm}$, and all objects were affixed to the floor of the apparatus with Blu Tack (Bostik, Stafford, UK) to prevent them from being displaced during a trial. As far as could be determined, the objects had no natural significance for the rats, and they had never been associated with a reinforcer.

General procedure. All rats were habituated in two consecutive daily sessions in which they were allowed to explore the empty Y-shaped apparatus for $5 \mathrm{~min}$. Before being placed in the apparatus, rats experienced a mock infusion procedure, as described above. After the mock infusion, the rats were taken from the preparation area to the testing room and placed in the start box; the guillotine door was then opened to allow the rat to explore the main area of the apparatus. When the rat exited the start box, the guillotine door was lowered to prevent re-entry into this area of the apparatus. The experimenter did not begin timing the session until after the rat exited the start box. Testing began $24 \mathrm{~h}$ after the second habituation session. At the start of each day, rats were transported to holding cages in a room adjacent to the main testing area. The rats remained in these holding cages throughout the day, except when they were being tested in the Y-shaped apparatus or receiving infusions. When all behavioral testing for that day was complete, the rats were returned to their home cages and fed. Rats were given a series of test trials, with a minimum interval of $24 \mathrm{~h}$ between trials. A different object pair was used for each trial for a given animal, and the order of exposure to object pairs as well as the designated sample and novel objects for each pair were counterbalanced within and across groups. The time spent exploring objects was assessed from video recordings of the sample and choice phases. Data were collected by scoring exploratory bouts using a personal computer running a program written in QuickBASIC 4.5. All trials were run with the experimenter blind to the drug treatment conditions.

Object recognition test. Each trial consisted of two phases. In the sample phase, two identical objects (A1 and A2) were placed in the Y-shaped apparatus, one at the end of each exploration arm. The rat was placed in the start box with the guillotine door lowered. The guillotine door was then raised to allow the rat into the exploration area of the maze. When the rat exited the start box, the guillotine door was lowered to prevent re-entry, and the sample phase began. The time spent exploring the two objects was scored by an experimenter viewing the rat on a video screen. 
The cumulative duration of exploratory bouts, the beginning and end of which were indicated by pressing a given key on the computer keyboard, was calculated by the computer program. Exploration of an object was defined as directing the nose to the object at a distance of $<2 \mathrm{~cm}$ and/or touching it with the nose. The sample phase ended when the rat had explored the identical objects for a total of $25 \mathrm{~s}$.

At the end of the sample phase, the rat was removed from the $\mathrm{Y}$-shaped apparatus for the duration of the retention delay, which was $24 \mathrm{~h}$ for all experiments. After the delay, the rat was placed back in the start box of the Y-shaped apparatus and released into the exploration area for the choice phase. The Y-shaped apparatus now contained an identical copy of the sample (familiar) object (A3) in one arm and a new object (B) in the other. The exploration arms in which the choice objects were placed were counterbalanced between rats and across trials. The rat was allowed to explore the objects for $2 \mathrm{~min}$, at the end of which it was removed and returned to its home cage. The time spent exploring the novel and familiar objects was recorded for the 2 min of the choice phase, but attention was focused on the first minute, during which object discrimination is typically greatest. We calculated a discrimination ratio, the proportion of total exploration time spent exploring the novel object (i.e., the difference in time spent exploring the novel and familiar objects divided by the total time spent exploring the objects), for the first minute of the choice phase on each object recognition trial. This measure takes into account individual differences in the total amount of exploration time.

Experiment 1. Experiment 1 assessed the effects of intra-PRh infusions of scopolamine in three groups of rats, whose infusion times corresponded to discrete stages within an object recognition trial. (1) The presample group $(n=10)$ received infusions, as described above, before the sample phase. Animals were placed into the $Y$-shaped apparatus $\sim 15$ min after the end of the infusion (disengagement of the infusion pump). (2) The postsample group $(n=9)$ received infusions immediately after the sample phase. After completion of the requisite $25 \mathrm{~s}$ of sample object exploration, the rat was removed from the apparatus and immediately transported to the infusion area for the start of intra-PRh infusions. After infusions, the rats were returned to the holding cages. (3) The prechoice group $(n=8)$ received infusions before the choice phase. Animals were placed into the Y-shaped apparatus for choice testing $\sim 15$ min after the end of the infusion. All rats were run for a total of four trials, two with saline infusions and two with scopolamine infusions. The order of drug and saline infusions was counterbalanced within and between groups.

Experiment 2. Experiment 2 was designed to examine further the effects of postsample infusions using three groups. (1) The immediate group $(n=10)$ received infusions of either saline or scopolamine immediately after the sample phase, as described for the postsample group in experiment 1. (2) The $20 \mathrm{~min}$ group $(n=9)$ received infusions $20 \mathrm{~min}$ after the end of the sample phase. At the end of the sample phase, these rats were transported to the individual holding cages until $20 \mathrm{~min}$ had passed. They were then given intra-PRh infusions of saline or scopolamine and returned to the holding cages for the remainder of the testing day. (3) The 40 min group $(n=8)$ underwent the same procedure as the 20 min group, except that the infusions were delivered at $40 \mathrm{~min}$ after the end of the sample phase. Each of the three groups was tested on four trials, two with saline and two with scopolamine, and the order of drug and saline infusions was counterbalanced within and between groups.

Experiment 3 . To verify and extend the effects of postsample infusions observed in experiment 2, four groups of animals were tested. (1) The immediate group $(n=8)$ received infusions of either saline or scopolamine immediately after the sample phase, as described for the postsample group in experiment 1. (2) The $8 \mathrm{~h}$ group $(n=8)$ received infusions $8 \mathrm{~h}$ after the sample phase. (3) The $16 \mathrm{~h}$ group $(n=8)$ received infusions $16 \mathrm{~h}$ after the sample phase. (4) The $20 \mathrm{~h}$ group $(n=7)$ received infusions $20 \mathrm{~h}$ after the end of the sample phase, $4 \mathrm{~h}$ before the choice phase. Each group was tested on four trials, two with saline and two with scopolamine, and the order of drug infusions was counterbalanced within and between groups.

Experiment 4. Experiment 4 compared the effects of immediate postsample infusions of saline or scopolamine to those of a control condition in which no infusions were delivered. This experiment was designed to test the hypothesis that the salience of the postsample infusion episode causes retroactive interference with the sample trace on saline trials and that postsample scopolamine facilitates performance by blocking this interference. Each of 12 rats was run for six trials in counterbalanced order. Each rat experienced two trials in each of the following three conditions: (1) immediate postsample saline, (2) immediate postsample scopolamine (the parameters of the saline and scopolamine trials were identical to those used for the postsample group in experiment 1), and (3) no infusion (on these trials, rats were removed from the Y-shaped apparatus immediately after the end of the sample phase and placed back into the individual holding cages with no additional handling). The noinfusion condition, thus, eliminated all aspects of handling and arousal associated with the infusion procedure.

Experiment 5. Experiment 5 compared the effects of $3 \mathrm{~h}$ presample infusions of saline or scopolamine to those of a control condition in which no infusions were delivered. This experiment was designed to test whether the detrimental retroactive effects of the infusion episode found in experiment 4 could also be demonstrated to operate in a proactive manner and, if so, whether scopolamine could block this impairment. Each of 10 rats was run for six trials in counterbalanced order. Each rat experienced two trials in each of the following three conditions: (1) $3 \mathrm{~h}$ presample saline, (2) $3 \mathrm{~h}$ presample scopolamine (in the saline and scopolamine conditions, infusions were delivered in the usual manner, but $3 \mathrm{~h}$ before the start of the sample phase), and (3) no infusion (on these trials, rats remained in the individual holding cages with no further handling until the sample phase). As in experiment 4 , the goal of this condition was to prevent the handling and arousal associated with the infusion episode.

Figure 1 illustrates the basic protocols used for each of the five experiments described above.

\section{Data analysis}

Group means of three measures taken from object recognition testing (duration of the sample phase, total exploration time in the choice phase, and the discrimination ratio) were analyzed for each experiment. Means from these measures were submitted to two-way ANOVA with repeated measures for experiments 1-3 (group by drug) and univariate repeatedmeasures ANOVAs for experiments 4 and 5. Paired-sample $t$ tests were used for post hoc analyses of within-subject effects, and Student-Newman-Keuls was used to analyze between-subject effects. All statistical analyses were conducted with a significance level of $\alpha=0.05$.

\section{Results}

\section{Cannula placements}

Rats in all experiments had cannulas implanted bilaterally within the PRh to allow delivery of drugs. All rats included in the behavioral analyses had guide cannulas located bilaterally with injection needle tips terminating in $\mathrm{PRh}$ near the border between areas 35 and 36 within cortical layers $2-5$ (Burwell, 2001). These placements were consistently located between 5.80 and $6.30 \mathrm{~mm}$ posterior to bregma (Fig. 2), the approximate midsection of the rostral-caudal extent of the PRh.

\section{Experiment 1: muscarinic receptor blockade during encoding, storage, and retrieval of object recognition memory}

We infused saline or scopolamine into the PRh 15 min before the sample phase, immediately after the sample phase, or $15 \mathrm{~min}$ before the choice phase to assess the effects of cholinergic muscarinic receptor blockade on encoding, storage, and retrieval, respectively. The total time required to meet the sample object exploration criterion ( 25 s cumulative) was analyzed, because a group difference at this stage of the trial might influence subsequent recognition performance. This analysis revealed no significant effects of group $\left(F_{(2,24)}=1.59\right)$, drug $\left(F_{(1,24)}=1.13\right)$, or the group by drug interaction term $(F<1)$. The means $( \pm$ SEM) for separate conditions were as follows: presample, saline, $88.89 \pm$ $16.06 \mathrm{~s}$, scopolamine, $89.87 \pm 11.29 \mathrm{~s}$; postsample, saline, $116.91 \pm 27.14 \mathrm{~s}$, scopolamine, $87.91 \pm 7.81 \mathrm{~s}$; prechoice, saline, 


\section{Sample Phase}

Retention Delay $(24 \mathrm{~h})$
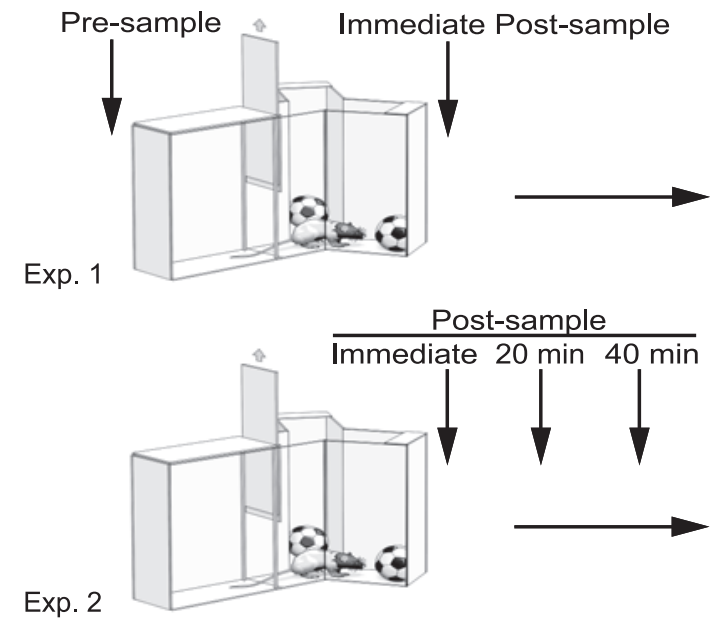

Exp. 2

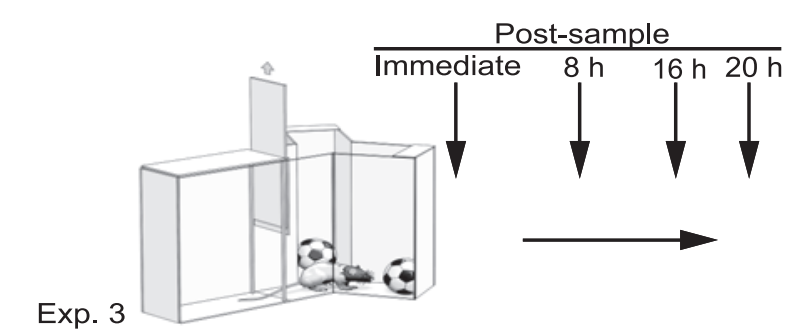

Exp. 3
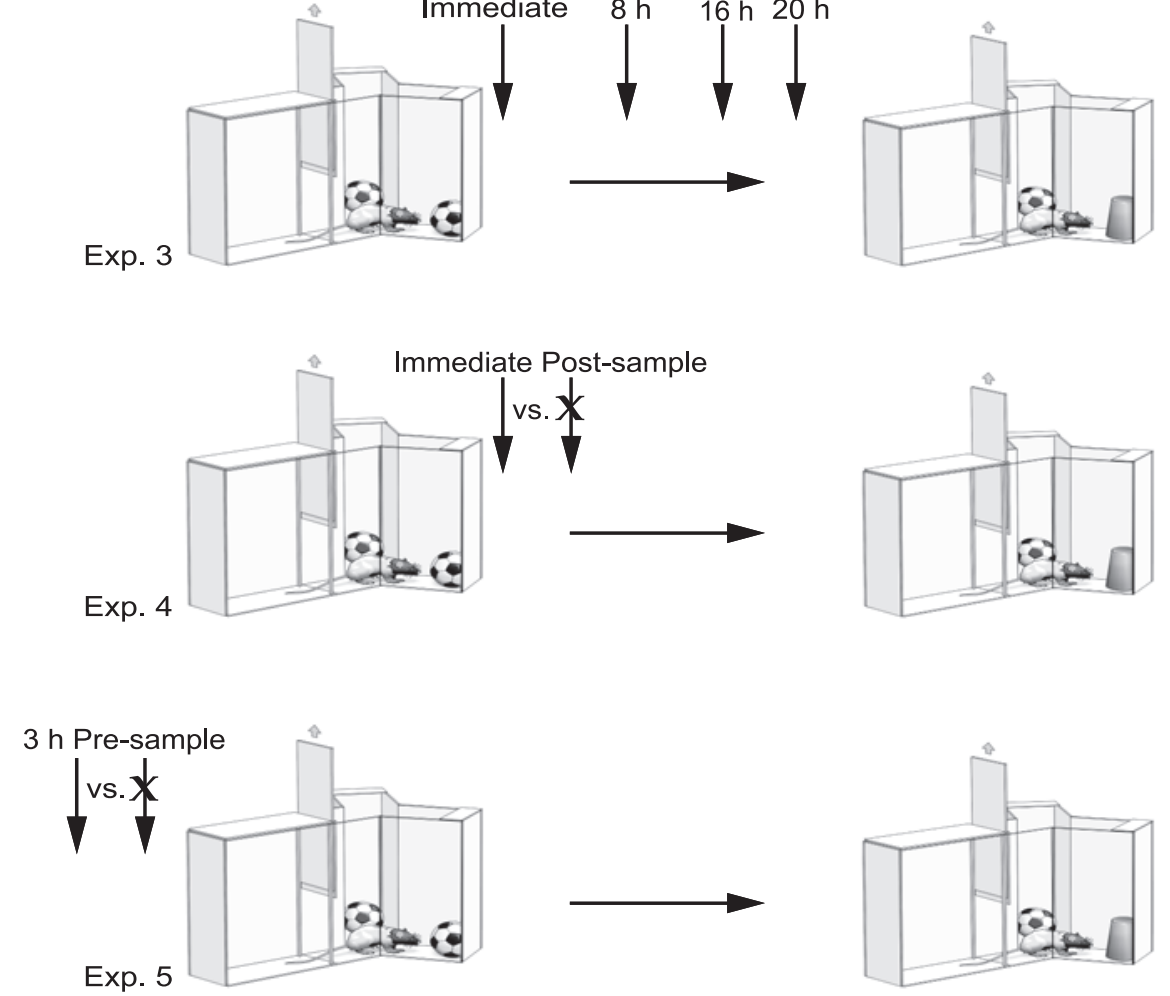

Figure 1. Schematic representation of the infusion protocols for each of the five experiments in the present study. The sample and choice phases are illustrated for each experiment (Exp.), and the vertical arrows indicate the various infusion times. In each experiment, a given object-recognition trial consisted of a sample phase in which the rat explored two identical objects, a $24 \mathrm{~h}$ retention delay, and a choice phase in which the rat was presented with a novel object and a triplicate copy of the sample object. Each vertical arrow indicates a group or condition in which saline and scopolamine were administered in counterbalanced manner over several trials in a given experiment. For experiments 4 and 5 , a vertical arrow with an $X$ represents the no-infusion condition, which was compared with the effects of immediate postsample or $3 \mathrm{~h}$ presample infusions of saline and scopolamine. The separation of time points in the figure is not to scale.

$76.49 \pm 9.38 \mathrm{~s}$, scopolamine, $70.26 \pm 9.58 \mathrm{~s}$. There was also no significant effect of group $\left(F_{(2,24)}=2.68\right)$, drug $(F<1)$, or group by drug interaction $(F<1)$ on the total amount of time spent by rats exploring the objects in the choice phase (means \pm SEM: presample, saline, $20.61 \pm 1.57 \mathrm{~s}$, scopolamine, $20.99 \pm 1.43 \mathrm{~s}$; postsample, saline, $19.09 \pm 1.41 \mathrm{~s}$, scopolamine, $16.79 \pm 1.98 \mathrm{~s}$; prechoice, saline, $21.84 \pm 1.58 \mathrm{~s}$, scopolamine, $19.81 \pm 1.94 \mathrm{~s})$. In contrast, intra$\mathrm{PRh}$ infusions of scopolamine had a significant effect on object recognition performance, which was related to the time at which the infusions were administered (Fig. 3); this was reflected in a significant group by drug interaction effect on the discrimination ratio scores $\left(F_{(2,24)}=\right.$ $6.28 ; p<0.01)$. Post hoc analysis with separate paired-sample $t$ tests revealed significant differences between saline and scopolamine trial performance in the presample $\left(t_{(9)}=2.25 ; p<0.05\right)$ and postsample $\left(t_{(8)}=2.58 ; p<0.05\right)$ groups, but not the prechoice group $(t<1)$. The main effects of group $\left(F_{(2,24)}=1.08\right)$ and drug $(F<1)$ were, however, not significant. Figure 3 shows that the performance of the presample group on scopolamine trials was significantly worse than on saline trials, whereas the opposite pattern was observed in the postsample group. Thus, presample injections of scopolamine impaired object recognition memory, prechoice injections had no effect, and postsample injections produced a facilitation of performance.

\section{Experiment 2: additional analysis of muscarinic receptor blockade during storage}

The results from the presample group in experiment 1 implicate PRh muscarinic cholinergic receptors in the encoding stage of object recognition memory. This finding is consistent with other recent reports of the effects of presample or pretraining intra-PRh scopolamine in object recognition tasks (Tang et al., 1997; Warburton et al., 2003). Our finding, however, that immediate postsample intra-PRh scopolamine facilitated object recognition performance was somewhat surprising. We have reported previously that transient inactivation of $\mathrm{PRh}$ with lidocaine up to, but not beyond, 20 min postsample blocked consolidation in the spontaneous object recognition task (Winters and Bussey, 2005a), and a similar postsample period of sensitivity to AMPA and NMDA glutamate receptor blockade also exists (Winters and Bussey, 2005c). We therefore sought to determine whether the facilitative effect of postsample muscarinic receptor blockade in experiment 1 might be the result of a direct action of scopolamine on the consolidation process suggested by these earlier results. Accordingly, for experiment 2 we conducted infusions of saline or scopolamine either immediately, $20 \mathrm{~min}$, or $40 \mathrm{~min}$ after the sample phase to determine whether a "window of facilitation" similar in time course to the detrimental effects of lidocaine and glutamate receptor antagonists could be observed. 


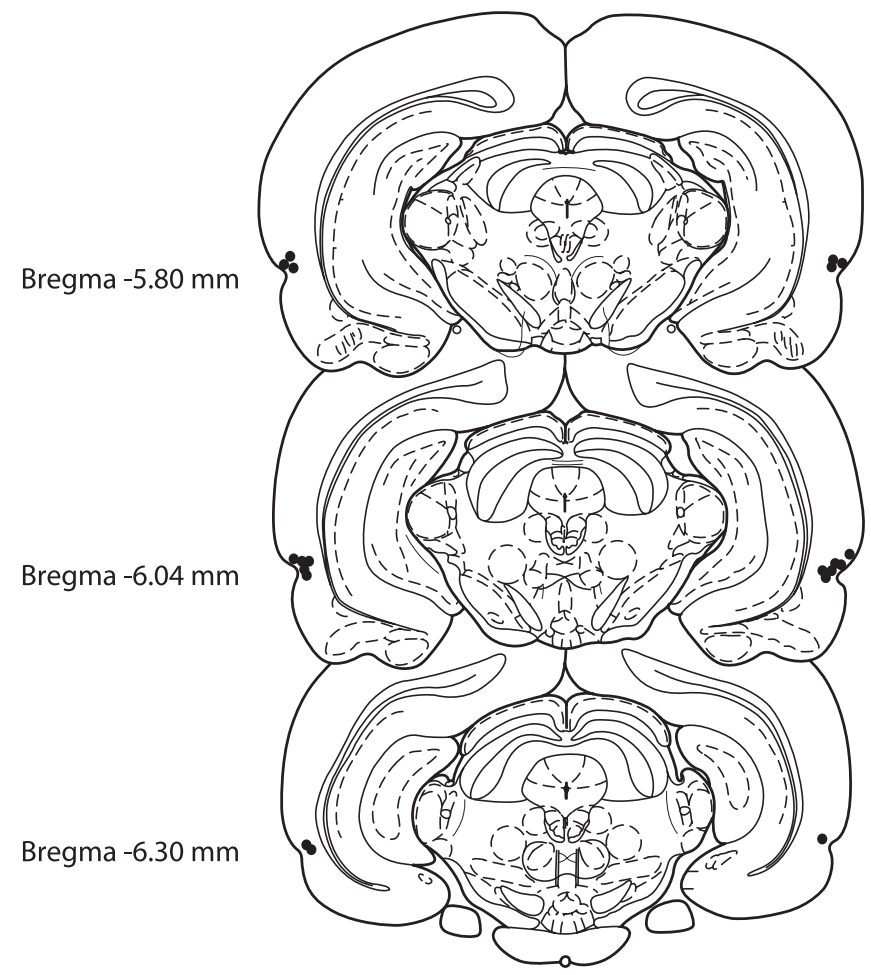

Figure 2. Cannulation of rat PRh. Schematic representation of the infusion needle tip placements from a typical group of animals (experiment 1; presample group; $n=10$ ). These placements are representative of needle tip locations in all animals included in the behavioral analyses of the present study. Cannulas were consistently located between 5.80 and $6.30 \mathrm{~mm}$ posterior to bregma. Some needle tips overlap in the figure.

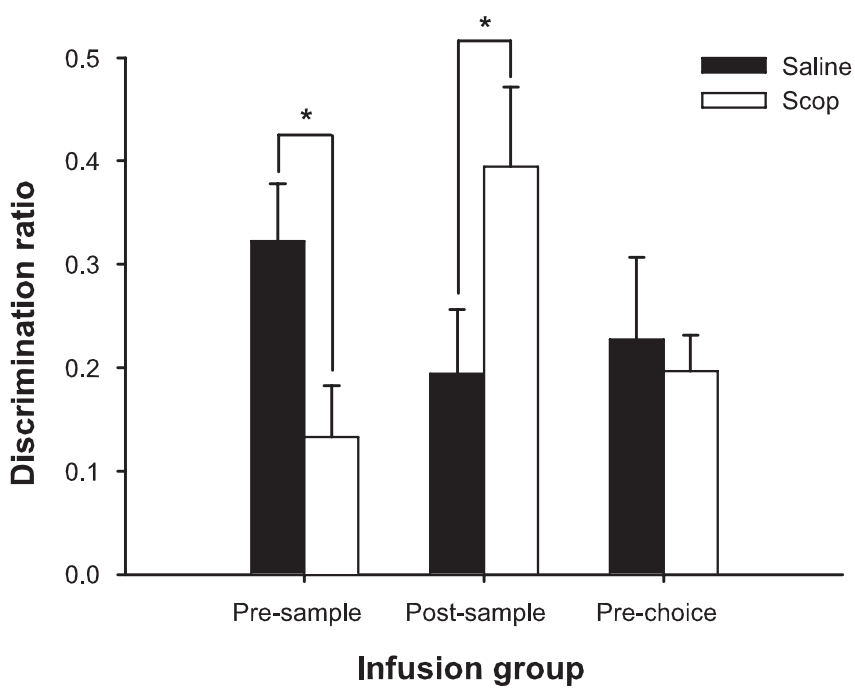

Figure 3. Spontaneous object recognition performance by animals in experiment 1 on trials in which they received bilateral intra-PRh infusions of scopolamine (Scop) or saline $15 \mathrm{~min}$ before the sample phase (presample), immediately after the sample phase (postsample), or 15 min before the choice phase (prechoice). The retention delay was $24 \mathrm{~h}$. Presample intra-PRh infusions of scopolamine disrupted object recognition, but postsample infusions facilitated memory relative to saline trials. Prechoice group performance did not differ between scopolamine and saline trials. Data are presented as average discrimination ratio \pm SEM. ${ }^{*} p<0.05$.

Analysis of the sample phase duration revealed no significant effects of the group $\left(F_{(2,24)}=2.34\right)$, drug $\left(F_{(1,24)}=2.79\right)$, or the group by drug interaction $(F<1)$ terms. The means $( \pm$ SEM) for separate conditions were as follows: immediate, saline, $114.96 \pm 12.77 \mathrm{~s}$, scopolamine, $93.83 \pm 10.31 \mathrm{~s} ; 20 \mathrm{~min}$,

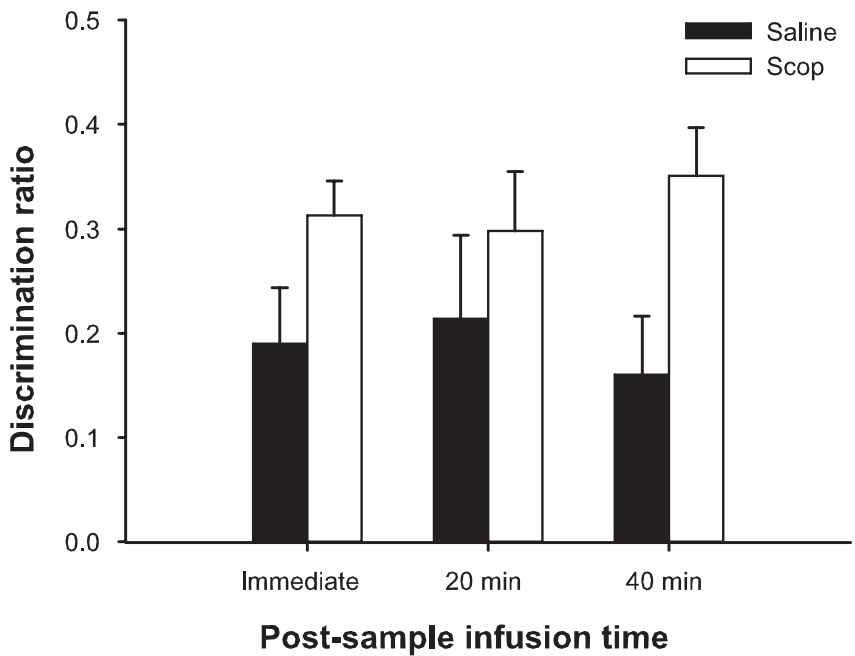

Figure 4. Spontaneous object recognition performance of the immediate, $20 \mathrm{~min}$, and 40 min postsample infusion groups in experiment 2 on trials in which they received either saline or scopolamine (Scop) infusions bilaterally into PRh. The retention delay was $24 \mathrm{~h}$. Scopolamine had an overall facilitating effect on object recognition memory. Data are presented as average discrimination ratio $\pm S E M$.

saline, $87.18 \pm 6.47 \mathrm{~s}$, scopolamine, $81.58 \pm 15.26 \mathrm{~s} ; 40 \mathrm{~min}$, saline, $84.93 \pm 8.86 \mathrm{~s}$, scopolamine, $76.15 \pm 9.33 \mathrm{~s}$. The analysis of total object exploration in the choice phase revealed significant group $\left(F_{(2,24)}=10.95 ; p<0.001\right)$ and group by drug $\left(F_{(2,24)}=\right.$ 3.61; $p<0.05)$ effects, as the immediate group explored less overall compared with the 20 and 40 min groups (means \pm SEM: immediate, saline, $17.48 \pm 1.34 \mathrm{~s}$, scopolamine, $13.57 \pm 0.85 \mathrm{~s} ; 20$ $\mathrm{min}$, saline, $22.29 \pm 1.01 \mathrm{~s}$, scopolamine, $21.09 \pm 2.05 \mathrm{~s} ; 40 \mathrm{~min}$, saline, $19.49 \pm 1.17 \mathrm{~s}$, scopolamine, $22.08 \pm 1.86 \mathrm{~s}$ ). Importantly, however, the main effect of drug on overall object exploration in the choice phase was not significant $(F<1)$. Conversely, there was a significant drug effect on object recognition performance $\left(F_{1,24)}=9.43 ; p<0.01\right)$, as scopolamine improved recognition memory relative to saline in all infusion groups (Fig. 4). The group and group by drug terms were, however, not significant (both $F<1$ ). Thus, postsample scopolamine facilitated object recognition memory, even with a 40 min interval between the end of the sample phase and the infusion.

Experiment 3: does muscarinic receptor blockade at even very long postsample intervals facilitate object recognition memory?

The findings with postsample intra-PRh scopolamine infusions from experiments 1 and 2 indicate that activation of muscarinic receptors in PRh during the retention delay can influence object recognition for stimuli previously encountered. Moreover, the results of experiment 2 reveal that the facilitative effect of postsample intra-PRh scopolamine may result from influencing a process other than the direct consolidation of the sample object trace, as previous findings have indicated that the sample trace may be fully consolidated between 20 and $40 \mathrm{~min}$ after the sample phase in the spontaneous object recognition paradigm (Winters and Bussey, 2005a,c). In contrast to reports that the detrimental effects of postsample intra-PRh lidocaine or glutamate receptor antagonists are no longer seen at a $40 \mathrm{~min}$ postsample interval, intra-PRh scopolamine improved memory for a previously sampled object regardless of whether the infusions were performed immediately, $20 \mathrm{~min}$, or $40 \mathrm{~min}$ postsample in experiment 2 . At the very least, it seems that the effects of scopolamine at the 40 


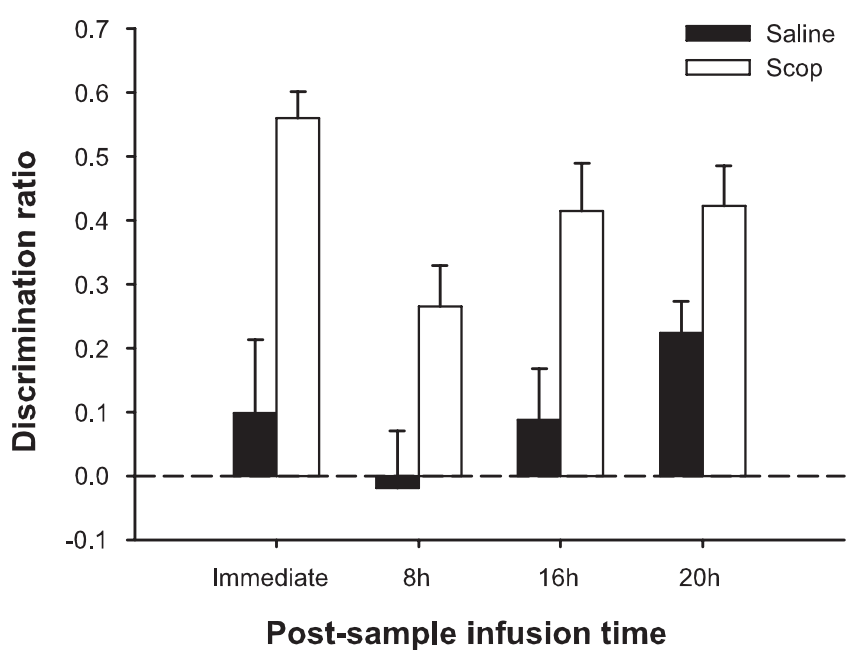

Figure 5. Spontaneous object recognition performance of the immediate, 8, 16, and $20 \mathrm{~h}$ postsample infusion groups in experiment 3 on trials in which they received either saline or scopolamine (Scop) infusions bilaterally into PRh. The retention delay was $24 \mathrm{~h}$. As in experiment 2, scopolamine had an overall facilitating effect on object recognition memory when infused after the sample phase. Data are presented as average discrimination ratio \pm SEM.

min postsample interval do not result from a direct effect on a consolidation process in PRh. In experiment 3, we therefore asked how long the postsample infusion of scopolamine could be delayed and still produce object recognition facilitation. Accordingly, we conducted intra-PRh infusions of saline or scopolamine immediately, 8,16 , or $20 \mathrm{~h}$ after the sample phase.

Although scopolamine did not affect measures of general object exploration, intra-PRh infusions produced a significant overall improvement in object recognition performance compared with saline trials (Fig. 5). Analysis of the total time required to explore the sample objects for $25 \mathrm{~s}$ revealed no significant effects of group $(F<1)$, drug $(F<1)$, or group by drug interaction $\left(F_{(3,28)}=1.81\right)$. The means $( \pm$ SEM $)$ for each group under each drug condition were as follows: immediate, saline, $80.46 \pm 12.81$ s, scopolamine, $75.99 \pm 7.25 \mathrm{~s} ; 8 \mathrm{~h}$, saline, $99.15 \pm 16.29 \mathrm{~s}$, scopolamine, $75.97 \pm 9.51 \mathrm{~s} ; 16 \mathrm{~h}$, saline, $69.41 \pm 6.77 \mathrm{~s}$, scopolamine, $87.86 \pm 10.79 \mathrm{~s} ; 20 \mathrm{~h}$, saline, $72.66 \pm 12.42 \mathrm{~s}$, scopolamine, $82.5 \pm 14.99 \mathrm{~s}$. The group $\left(F_{(3,28)}=1.48\right)$, drug $(F<1)$, and interaction $\left(F_{(3,28)}=1.34\right)$ terms also failed to reach significance when the total object exploration time in the choice phase was analyzed (means \pm SEM: immediate, saline, $17.93 \pm 1.8 \mathrm{~s}$, scopolamine, $15.17 \pm 1.39 \mathrm{~s} ; 8 \mathrm{~h}$, saline, $18.73 \pm 1.84 \mathrm{~s}$, scopolamine, $20.66 \pm 1.74 \mathrm{~s} ; 16 \mathrm{~h}$, saline, $18.29 \pm 1.18 \mathrm{~s}$, scopolamine, $18.95 \pm 1.73 \mathrm{~s} ; 20 \mathrm{~h}$, saline, $19.73 \pm 1.03 \mathrm{~s}$, scopolamine, $20.04 \pm$ $2.05 \mathrm{~s})$. Analysis of the discrimination ratios for object recognition, however, revealed significant main effects of drug $\left(F_{(1,28)}=\right.$ 40.39; $p<0.001)$ and group $\left(F_{(3,28)}=3.8 ; p<0.05\right)$. Post hoc Newman-Keuls analysis of the latter effect indicated that the $8 \mathrm{~h}$ infusion group performed significantly worse overall than the immediate and $20 \mathrm{~h}$ (both $p<0.05$ ), but not the $16 \mathrm{~h}$, infusion groups. The group by drug interaction term was not significant $\left(F_{(3,28)}=1.19\right)$. Indeed, Figure 5 shows that object recognition performance with scopolamine infusions was better at all postsample infusion intervals when compared with performance on saline infusion trials.

\section{Experiment 4: does scopolamine block the acquisition of interfering information?}

A notable aspect of the postsample infusion results from experiments $1-3$ is the fact that performance on saline trials generally

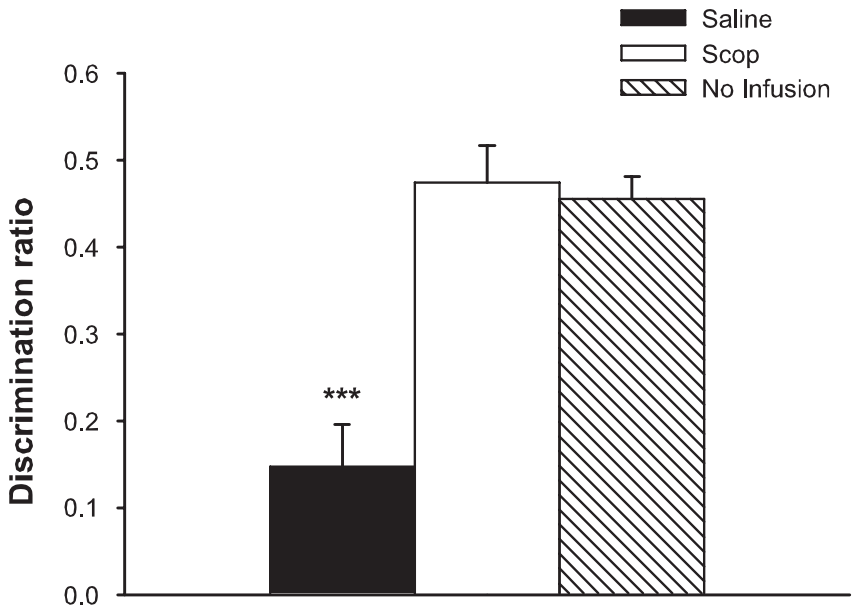

Figure 6. Spontaneous object recognition performance of rats in experiment 4 on trials in which they received intra-PRh saline, intra-PRh scopolamine (Scop), or no infusion immediately after the sample phase. The retention delay was $24 \mathrm{~h}$. Object recognition on saline trials was disrupted compared with memory performance in the scopolamine and no-infusion conditions. Data are presented as average discrimination ratio \pm SEM. ${ }^{* * *} p<0.001$, saline versus scopolamine and saline versus no infusion.

seemed to be compromised throughout [compare the performance of saline-infused animals with control animals in the studies by Winters et al. (2004) and Forwood et al. (2005)]. It seems that, rather than directly facilitating memory per se, intra-PRh scopolamine reversed or blocked some process that interfered with normal object recognition in the saline infusion conditions. We hypothesized that the facilitation caused by postsample scopolamine could be the result of impaired acquisition of information that might interfere retroactively with the sample object trace. Accordingly, we speculated that arousal associated with the infusion episode might encourage encoding of information that could interfere with subsequent object recognition memory performance. According to this view, the scopolamine-induced facilitation could be because of scopolamine blocking the encoding of this interfering information in the same way that presample infusions of scopolamine were seen to disrupt encoding of the sample object in experiment 1 . The hypothesis that the infusion episode is somehow directly linked to the poor performance on postsample saline trials is consistent with the findings that saline performance was generally poor at all postsample infusion times and the degree of memory amelioration with scopolamine was equivalent across all postsample conditions. To test this hypothesis, we compared the effects of immediate postsample intra-PRh infusions of scopolamine or saline with a third condition involving minimal postsample handling and no infusion. If the infusion episode does indeed result in some kind of retroactive interference that can be blocked by scopolamine, object recognition memory on trials with no infusions should be as good as it is on scopolamine trials.

There was no difference between the three conditions in the time taken to explore the objects in the sample phase $(F<1$; means \pm SEM: saline, $73.46 \pm 6.24 \mathrm{~s}$; scopolamine, $88.55 \pm$ $13.6 \mathrm{~s}$; no infusion, $90.15 \pm 12.23 \mathrm{~s}$ ) or the total exploration time in the choice phase $(F<1$; means \pm SEM: saline, $17.47 \pm 1.33 \mathrm{~s}$; scopolamine, $15.51 \pm 1.27 \mathrm{~s}$; no infusion, $16.16 \pm 1.24 \mathrm{~s})$. Intra$\mathrm{PRh}$ infusions of saline, however, significantly disrupted object recognition memory compared with trials on which animals received scopolamine or no infusions (Fig. 6). A univariate ANOVA on the discrimination ratio scores revealed a significant 
effect of condition $\left(F_{(2,22)}=23.07 ; p<0.001\right)$. Post hoc comparisons indicated that performance on saline trials differed significantly from scopolamine $\left(t_{(11)}=6.53 ; p<0.001\right)$ and no-infusion $\left(t_{(11)}=5.23 ; p<0.001\right)$ trials, whereas object recognition on no-infusion trials was similar to that with scopolamine $(t<1)$. Thus, immediate postsample saline infusions disrupted object recognition memory, and omitting the infusion episode, or infusing scopolamine, preserved memory performance.

\section{Experiment 5: can the infusion episode cause proactive interference and, if so, can scopolamine block it?}

The results of experiment 4 highlight an important practical consideration for experiments in which the effects of postacquisition manipulations on learning and memory are studied. The disparate effects of the saline and no-infusion conditions indicate that the physical act of infusing or injecting drugs can have consequences for memory performance, at least under challenging conditions such as the $24 \mathrm{~h}$ retention delay used in the present study. Indeed, the results of experiment 4 support our hypothesis that some aspect of the infusion episode contributed to the poor performance seen in postsample saline infusion conditions throughout the present study; merely omitting the infusion was sufficient to recover object recognition memory to the same levels as those seen on scopolamine trials. Our suggestion is that postsample scopolamine facilitates object recognition memory relative to saline by blocking the encoding of interfering information at or around the time of the infusion. This hypothesis is intuitively appealing because it implies that scopolamine may disrupt or facilitate memory through the same process (blockade of acquisition) depending on the target information and the context within which the drug is administered. Nonetheless, although it appears that the effects of scopolamine were not caused by influencing a consolidation process in the PRh, the possibility remains that scopolamine may have in some other way directly affected the processing of the sample stimulus to enhance storage during the retention delay and thereby facilitate recognition. In experiment 5, we tested the possibility that the same kind of interference we have hypothesized to be caused by postsample infusions in the previous studies might also operate proactively to disrupt object recognition and that this, too, could be blocked by intra-PRh infusions of scopolamine. Were this to be the case, it would strongly support our hypothesis that postsample scopolamine facilitates recognition memory by blocking acquisition of interfering information and would be inconsistent with a direct influence of scopolamine on sample processing, storage, or consolidation during the retention interval. We therefore compared the effects of intra-PRh infusions of saline or scopolamine given $3 \mathrm{~h}$ before the sample phase with those of a condition in which no presample infusions were delivered. We predicted that a $3 \mathrm{~h}$ presample infusion would allow sufficient time for the effects of scopolamine to dissipate such that there would not be any direct impairment of acquisition during the sample phase.

The three conditions did not differentially affect the time taken to explore the sample objects $(F<1$; means \pm SEM: saline, $102.91 \pm 6.26 \mathrm{~s}$; scopolamine, $92.89 \pm 9.58 \mathrm{~s}$; no infusion, $95.17 \pm 9.51 \mathrm{~s})$ or the total object exploration time in the choice phase $(F<1$; means \pm SEM: saline, $15.69 \pm 1.19 \mathrm{~s}$; scopolamine, $17.15 \pm 1.7 \mathrm{~s}$; no infusion, $15.78 \pm 1.6 \mathrm{~s})$. The analysis of the discrimination ratios, however, yielded a significant effect of condition $\left(F_{(2,18)}=11.78 ; p<0.01\right)$ (Figure 7 ), as object recognition memory on saline trials was significantly impaired relative to both scopolamine $\left(t_{(9)}=5 ; p<0.01\right)$ and no-infusion trials

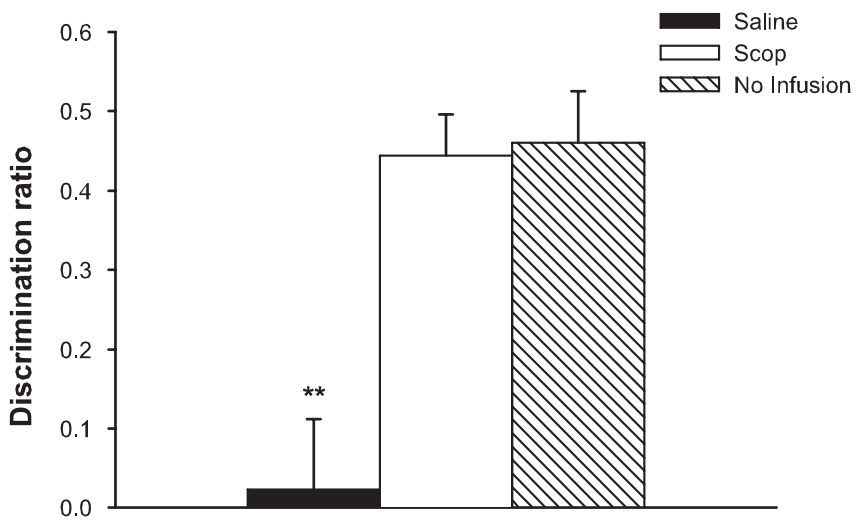

Figure 7. Spontaneous object recognition performance of rats in experiment 5 on trials in which they received intra-PRh saline, intra-PRh scopolamine ( $S c o p)$, or no infusion $3 \mathrm{~h}$ before the sample phase. The retention delay was $24 \mathrm{~h}$. Object recognition on saline trials was disrupted compared with memory performance in the scopolamine and no-infusion conditions. Data are presented as average discrimination ratio \pm SEM. ${ }^{* *} p<0.01$, saline versus scopolamine and saline versus no infusion.

$\left(t_{(9)}=3.41 ; p<0.01\right)$. Performance in the scopolamine and no-infusion conditions did not differ $(t<1)$. Thus, the infusion episode can cause interference retroactively and proactively. This interference is sufficient to disrupt object recognition memory with a relatively long retention delay $(24 \mathrm{~h})$ and can be blocked by antagonism of muscarinic receptors in PRh, which reverses the memory deficit.

\section{Discussion}

The present results suggest that cortical ACh facilitates acquisition of new declarative information rather than directly influencing the storage or consolidation process in the absence of the to-be-remembered stimulus material. Presample intra-PRh infusions of scopolamine significantly disrupted object recognition memory with a $24 \mathrm{~h}$ retention delay. Prechoice infusions with the same parameters had no obvious effect on memory retrieval. Infusions during the retention delay, however, significantly improved object recognition compared with performance on trials with saline infusions. This facilitation of memory performance by intra-PRh scopolamine was very reliable and was observed with even very long sample-to-infusion intervals $(20 \mathrm{~h})$. The relatively poor performance on postsample and $3 \mathrm{~h}$ presample saline infusion trials compared with trials with no infusion condition suggests that the act of infusing encourages interference with the object trace acquired during the sample phase. All of these behavioral effects of scopolamine may, therefore, be mediated by the same underlying mechanism; specifically, disruption of sample acquisition may cause subsequent recognition impairment when scopolamine is administered directly before the sample phase, whereas scopolamine infusions during the retention delay or $3 \mathrm{~h}$ before the sample phase may block the acquisition of potentially interfering information, thereby improving recognition memory.

\section{Intra-PRh scopolamine disrupts acquisition, but not retrieval, of object information}

A growing body of evidence indicates that preacquisition administration of scopolamine disrupts object recognition memory (Aigner and Mishkin, 1986; Huston and Aggleton, 1987; Ennaceur and Meliani, 1992; Dodart et al., 1997; Tang et al., 1997; Warburton et al., 2003), and permanent cholinergic denervation of PRh with 192 IgG-saporin in rats (Winters and Bussey, 2005b) 
and ME20.4-saporin in monkeys (Turchi et al., 2005) also impairs visual recognition. Consistent with these findings, presample, but not prechoice, intra-PRh infusions of scopolamine in the present study significantly impaired object recognition. These results indicate a role for $\mathrm{PRh} \mathrm{ACh}$ in the acquisition, but not retrieval, of object information. Accordingly, evidence from human studies suggests that increased cortical ACh can enhance the selectivity of perceptual processing in the extrastriate cortex during encoding in a visual working memory task (Furey et al., 1997, 2000), and scopolamine has been shown to reduce encodingrelated activity in the temporal lobes (including PRh) during visual delayed matching to sample (Schon et al., 2005). Moreover, reports indicate that direct application of cholinergic agents can fine-tune the receptive fields of cortical pyramidal cells (Krnjevic and Phillis, 1963; Sillito and Kemp, 1983; Murphy and Sillito, 1991; Rasmusson, 2000), and such findings have long been suggested as evidence for a role of cortical ACh in facilitating information acquisition, perhaps by amplifying the cortical processing of sensory stimuli (Hasselmo and Bower, 1993; Everitt and Robbins, 1997; Sarter and Bruno, 1997; Weinberger, 2003; Hasselmo and McGaughy, 2004). Thus, a local effect on stimulus acquisition within PRh might explain the impairment caused by presample scopolamine.

\section{Does intra-PRh scopolamine prevent the acquisition of interfering information?}

Unlike its presample effects, postsample infusions of scopolamine within the retention delay significantly improved object recognition memory compared with saline trials, and this was a highly replicable and robust effect. Indeed, memory facilitation was found even when the delay between the end of the sample phase and the infusion was as long as $20 \mathrm{~h}$. We posit that the facilitative effects of postsample scopolamine on object recognition were caused by a disruption of new information acquisition during storage of the sample trace, which minimized retroactive interference. It has been suggested, however, that low levels of cortical ACh encourage consolidation (Hasselmo, 1999; Hasselmo and McGaughy, 2004), and this is a possible alternative explanation for the postsample effects of scopolamine. However, we have previously presented data that suggest that consolidation within PRh is complete within $40 \mathrm{~min}$ of the end of the sample phase (Winters and Bussey, 2005a,c), yet scopolamine facilitated memory even when it was infused at 8,16 , or $20 \mathrm{~h}$ after the sample presentation. Moreover, the nature of the postsample effects suggests that scopolamine, rather than directly acting on the storage process to improve memory above and beyond stable control levels, instead blocked a detrimental outcome of the intradelay infusion procedure. Thus, performance on postsample saline infusion trials was poor throughout, whereas postsample scopolamine maintained performance at normally expected levels. Accordingly, the results of experiments 4 and 5 revealed that simply omitting the infusion episode returned performance to the level of scopolamine infusion trials. Interestingly, this effect was observed with $3 \mathrm{~h}$ presample and immediate postsample infusions, suggesting that the infusion episode leads to interference and that this interference can act either proactively or retroactively. In both cases, intra-PRh scopolamine blocked the detrimental effects of the infusion episode. The fact that $3 \mathrm{~h}$ presample infusions of scopolamine, unlike immediate presample administration, facilitated memory in an almost identical manner to postsample infusions is particularly interesting and strongly suggests that the postsample effects of scopolamine were not caused by a direct action on the consolidation, or indeed any other processing, of the sample object trace. These results are consistent with reports of normally amnesia-inducing drugs (e.g., alcohol, benzodiazepines) causing counterintuitive memory facilitation when they are administered after the acquisition of new to-be-remembered information (Wixted, 2004), and the interpretation of such findings in terms of attenuation of interfering information is similar to that suggested above.

The exact nature of the proposed interference produced by infusions remains unclear. It is possible that stress caused by the handling involved in the infusion procedure played a role in the deficit seen with postsample saline infusions. However, gentle handing was used during infusions, and rats were habituated to the procedure and did not ever appear to be stressed during infusions. Furthermore, a recent report indicates that, if anything, increased levels of glucocorticoids caused by stress shortly after the sample phase should improve object recognition memory (Okuda et al., 2004), which was not the case after saline infusions. Conceivably, infusions may produce interference by causing a general arousal in the animal that leads to the enhanced encoding of sensory information. It may be this sensory information that leads to interference with the sample trace. It is interesting to note that performance with postsample saline infusions in the present study was substantially poorer than in our previous studies (Winters and Bussey, 2005a,c). The current series of experiments is the first in which we have used a $24 \mathrm{~h}$ retention delay to assess the effects of postsample infusions. Previously, the longest delay we have used was $3 \mathrm{~h}$. The interference effect observed in the present study might only exert a significant influence on memory when the retention delay is relatively long. It would, therefore, be interesting to assess the effects of the present manipulations with shorter retention delays for comparison with the present results. On the basis of the present findings, however, regardless of the effects of postsample saline, we would predict that scopolamine would not disrupt memory consolidation with a shorter (e.g., $3 \mathrm{~h}$ ) retention delay.

There is growing evidence that ACh in PRh may directly influence synaptic plasticity related to object recognition memory (Massey et al., 2001; Bashir, 2003; Warburton et al., 2003), and such findings suggest a role for PRh ACh in consolidation and storage mechanisms. It would seem, however, that any effects of ACh on synaptic plasticity must occur in the very early stages of stimulus processing, because immediate postsample administration of scopolamine does not consistently disrupt object recognition memory (Aigner et al., 1991; Warburton et al., 2003) and, in fact, facilitated performance in the present study. This result contrasts with our previous findings that have indicated a role for other aspects of PRh transmission in object trace consolidation by showing that immediate postsample infusions of lidocaine or glutamate receptor antagonists severely impair object recognition memory (Winters and Bussey, 2005a,c) and argues strongly against the idea that scopolamine's effects were caused by a direct action on the consolidation process. It is possible, however, that an ACh-mediated enhancement of stimulus encoding and acquisition could increase the likelihood of successful consolidation and subsequent trace storage, augmenting any plastic changes associated with the acquisition episode. The present pattern of results, however, suggests that the primary effect of cortical ACh in declarative memory acquisition likely occurs when the stimulus material is present.

In contrast to the present findings, many previous studies have indicated that posttraining administration of cholinergic agents appear to affect memory consolidation (Power et al., 2003); for example, posttraining manipulations that reduce cho- 
linergic system efficacy disrupt memory in tasks such as fear conditioning, inhibitory avoidance, and conditioned place preference (Rudy, 1996; Bianchin et al., 1999; Power and McGaugh, 2002; Schroeder and Packard, 2002), whereas posttraining administration of muscarinic receptor agonists has been reported to improve memory in such tasks (Vazdarjanova and McGaugh, 1999; Farr et al., 2000). It is interesting to note that the vast majority of these effects are found in tasks that are thought to tax "emotional" memory and, therefore, are highly influenced by the amygdala (Power et al., 2003). Such results sit in stark contrast to our present findings suggesting an adverse effect of increased postacquisition ACh on object recognition, which is thought to tax declarative memory. This pattern indicates possibly different roles for ACh in amygdala-mediated emotional learning and declarative memory. Indeed, Gais and Born (2004a) have shown, consistent with recent suggestions by Hasselmo et al. (2004), that increasing ACh levels with physostigmine prevents the facilitative effects of postlearning sleep on declarative memory in humans; although it is unclear whether this effect was caused by similar mechanisms to those suggested by the present results, it nonetheless provides further indication that, particularly in the case of declarative memory, posttraining cortical ACh may not play a direct role in memory consolidation and can even be detrimental to memory.

The above discussion highlights a potentially important aspect of the role of ACh in learning and memory. There is strong evidence for a direct role for ACh in the consolidation of emotional associations such as those formed in fear conditioning, and this function of ACh may be closely linked to the involvement of the amygdala in such forms of learning and memory [but see Young et al. (1995), in which posttraining systemic administration of scopolamine facilitated fear conditioning to a tone. Learning about the discrete tone stimulus may have been interfered with by the effects of immediate posttraining injections, and scopolamine may have blocked this interference to facilitate memory by a process similar to the one we posit to explain the present results]. The present findings and others indicate that, although cortical ACh is vital for new information acquisition, it is not required for consolidation of declarative memory. Thus, it may be that, depending on the nature of the memory and the specific brain regions involved, different mechanisms mediate consolidation, and not all such mechanisms require ACh.

\section{Conclusion}

The findings of the present study indicate that disruption of cholinergic function in PRh can impair or facilitate object recognition memory depending on when this disruption takes place. We propose that a single underlying mechanism, impaired acquisition of new information, can explain these results. The pattern of presample and postsample effects together is inconsistent with a direct role for cortical ACh in declarative memory consolidation or other postsample stimulus processing. Nonetheless, it is likely that consolidation and storage processes are influenced by the direct enhancement by ACh of cortical information processing during acquisition.

\section{References}

Abe H, Ishida Y, Iwasaki T (2004) Perirhinal N-methyl-D-aspartate and muscarinic systems participate in object recognition in rats. Neurosci Lett 356:191-194.

Aigner TG, Mishkin M (1986) The effects of physostigmine and scopolamine on recognition memory in monkeys. Behav Neural Biol 45:81-87. Aigner TG, Walker DL, Mishkin M (1991) Comparison of the effects of scopolamine administered before and after acquisition in a test of visual recognition memory in monkeys. Behav Neural Biol 55:61-67.

Bartus RT (2000) On neurodegenerative diseases, models, and treatment strategies: lessons learned and lessons forgotten a generation following the cholinergic hypothesis. Exp Neurol 163:495-529.

Bartus RT, Dean RL, Pontecorvo MJ, Flicker C (1985) The cholinergic hypothesis: a historical overview, current perspective, and future directions. Ann NY Acad Sci 444:332-358.

Bashir ZI (2003) On long-term depression induced by activation of G-protein coupled receptors. Neurosci Res 45:363-367.

Bianchin M, Mello e Souza T, Medina JH, Izquierdo I (1999) The amygdala is involved in the modulation of long-term memory, but not in working or short-term memory. Neurobiol Learn Mem 71:127-131.

Bucci DJ, Holland PC, Gallagher M (1998) Removal of cholinergic input to rat posterior parietal cortex disrupts incremental processing of conditioned stimuli. J Neurosci 18:8038-8046.

Buffalo EA, Reber PJ, Squire LR (1998) The human perirhinal cortex and recognition memory. Hippocampus 8:330-339.

Burwell RD (2001) Borders and cytoarchitecture of the perirhinal and postrhinal cortices in the rat. J Comp Neurol 437:17-41.

Chiba AA, Bucci DJ, Holland PC, Gallagher M (1995) Basal forebrain cholinergic lesions disrupt increments but not decrements in conditioned stimulus processing. J Neurosci 15:7315-7322.

Dodart JC, Mathis C, Ungerer A (1997) Scopolamine-induced deficits in a two-trial object recognition task in mice. NeuroReport 8:1173-1178.

Ennaceur A, Delacour J (1988) A new one-trial test for neurobiological studies of memory in rats. I: Behavioral data. Behav Brain Res 31:47-59.

Ennaceur A, Meliani K (1992) Effects of physostigmine and scopolamine on rats' performances in object-recognition and radial-maze tests. Psychopharmacology (Berl) 109:321-330.

Everitt BJ, Robbins TW (1997) Central cholinergic systems and cognition. Annu Rev Psychol 48:649-684.

Farr SA, Uezu K, Creonte TA, Flood JF, Morley JE (2000) Modulation of memory processing in the cingulate cortex of mice. Pharmacol Biochem Behav 65:363-368.

Forwood SE, Winters BD, Bussey TJ (2005) Hippocampal lesions that abolish spatial maze performance spare object recognition memory at delays of up to 48 hours. Hippocampus 15:347-355.

Furey ML, Pietrini P, Haxby JV, Alexander GE, Lee HC, VanMeter J, Grady CL, Shetty U, Rapoport SI, Schapiro MB, Freo U (1997) Cholinergic stimulation alters performance and task-specific regional cerebral blood flow during working memory. Proc Natl Acad Sci USA 94:6512-6516.

Furey ML, Pietrini P, Haxby JV (2000) Cholinergic enhancement and increased selectivity of perceptual processing during working memory. Science 290:2315-2319.

Gais S, Born J (2004a) Low acetylcholine during slow-wave sleep is critical for declarative memory consolidation. Proc Natl Acad Sci USA 101:2140-2144.

Gais S, Born J (2004b) Declarative memory consolidation: mechanisms acting during human sleep. Learn Mem 11:679-685.

Hasselmo ME (1999) Neuromodulation: acetylcholine and memory consolidation. Trends Cogn Sci 3:351-359.

Hasselmo ME, Bower JM (1993) Acetylcholine and memory. Trends Neurosci 16:218-222.

Hasselmo ME, McGaughy J (2004) High acetylcholine levels set circuit dynamics for attention and encoding and low acetylcholine levels set dynamics for consolidation. Prog Brain Res 145:207-231.

Huston AE, Aggleton JP (1987) The effects of cholinergic drugs upon recognition memory in rats. Q J Exp Psychol B 39:297-314.

Krnjevic K, Phillis JW (1963) Acetylcholine-sensitive cells in the cerebral cortex. J Physiol 166:296-327.

Manns JR, Stark CE, Squire LR (2000) The visual paired-comparison task as a measure of declarative memory. Proc Natl Acad Sci USA 97:12375-12379.

Massey PV, Bhabra G, Cho K, Brown MW, Bashir ZI (2001) Activation of muscarinic receptors induces protein synthesis-dependent long-lasting depression in the perirhinal cortex. Eur J Neurosci 14:145-152.

McGaughy J, Koene RA, Eichenbaum H, Hasselmo ME (2005) Cholinergic deafferentation of the entorhinal cortex in rats impairs encoding of novel but not familiar stimuli in a delayed nonmatch-to-sample task. J Neurosci 25:10273-10281.

Meunier M, Bachevalier J, Mishkin M, Murray EA (1993) Effects on visual 
recognition of combined and separate ablations of the entorhinal and perirhinal cortex in rhesus monkeys. J Neurosci 13:5418-5432.

Morris RG, Kopelman MD (1986) The memory deficits in Alzheimer-type dementia: a review. Q J Exp Psychol A 38:575-602.

Mumby DG, Pinel JP (1994) Rhinal cortex lesions and object recognition in rats. Behav Neurosci 108:11-18.

Murphy PC, Sillito AM (1991) Cholinergic enhancement of direction selectivity in the visual cortex of the cat. Neuroscience 40:13-20.

Okuda S, Roozendaal B, McGaugh JL (2004) Glucocorticoid effects on object recognition memory require training-associated emotional arousal. Proc Natl Acad Sci USA 101:853-858.

Paxinos G, Watson C (1997) The rat brain in stereotaxic coordinates, Ed 3. London: Academic.

Perry EK, Tomlinson BE, Blessed G, Bergmann K, Gibson PH, Perry RH (1978) Correlation of cholinergic abnormalities with senile plaques and mental test scores in senile dementia. Br Med J 2:1457-1459.

Power AE, McGaugh JL (2002) Phthalic acid amygdalopetal lesion of the nucleus basalis magnocellularis induces reversible memory deficits in rats. Neurobiol Learn Mem 77:372-388.

Power AE, Vazdarjanova A, McGaugh JL (2003) Muscarinic cholinergic influences in memory consolidation. Neurobiol Learn Mem 80:178-193.

Rasmusson DD (2000) The role of acetylcholine in cortical synaptic plasticity. Behav Brain Res 115:205-218.

Rudy JW (1996) Scopolamine administered before and after training impairs both contextual and auditory-cue fear conditioning. Neurobiol Learn Mem 65:73-81.

Sarter M, Bruno JP (1997) Cognitive functions of cortical acetylcholine: toward a unifying hypothesis. Brain Res Brain Res Rev 23:28-46.

Sarter M, Bruno JP, Givens B (2003) Attentional functions of cortical cholinergic inputs: what does it mean for learning and memory? Neurobiol Learn Mem 80:245-256.

Schon K, Atri A, Hasselmo ME, Tricarico MD, LoPresti ML, Stern CE (2005) Scopolamine reduces persistent activity related to long-term encoding in the parahippocampal gyrus during delayed matching in humans. J Neurosci 25:9112-9123.

Schroeder JP, Packard MG (2002) Posttraining intra-basolateral amygdala scopolamine impairs food- and amphetamine-induced conditioned place preferences. Behav Neurosci 116:922-927.

Sillito AM, Kemp JA (1983) Cholinergic modulation of the functional organization of the cat visual cortex. Brain Res 289:143-155.
Tang Y, Mishkin M, Aigner TG (1997) Effects of muscarinic blockade in perirhinal cortex during visual recognition. Proc Natl Acad Sci USA 94:12667-12669.

Turchi J, Saunders RC, Mishkin M (2005) Effects of cholinergic deafferentation of the rhinal cortex on visual recognition memory in monkeys. Proc Natl Acad Sci USA 102:2158-2161.

Vazdarjanova A, McGaugh JL (1999) Basolateral amygdala is involved in modulating consolidation of memory for classical fear conditioning. J Neurosci 19:6615-6622.

Warburton EC, Koder T, Cho K, Massey PV, Duguid G, Barker GR, Aggleton JP, Bashir ZI, Brown MW (2003) Cholinergic neurotransmission is essential for perirhinal cortical plasticity and recognition memory. Neuron 38:987-996.

Weinberger NM (2003) The nucleus basalis and memory codes: auditory cortical plasticity and the induction of specific, associative behavioral memory. Neurobiol Learn Mem 80:268-284.

Winters BD, Bussey TJ (2005a) Transient inactivation of perirhinal cortex disrupts encoding, retrieval, and consolidation of object recognition memory. J Neurosci 25:52-61.

Winters BD, Bussey TJ (2005b) Removal of cholinergic input to perirhinal cortex disrupts object recognition but not spatial working memory in the rat. Eur J Neurosci 21:2263-2270.

Winters BD, Bussey TJ (2005c) Glutamate receptors in perirhinal cortex mediate encoding, retrieval, and consolidation of object recognition memory. J Neurosci 25:4243-4251.

Winters BD, Forwood SE, Cowell RA, Saksida LM, Bussey TJ (2004) Double dissociation between the effects of peri-postrhinal cortex and hippocampal lesions on tests of object recognition and spatial memory: heterogeneity of function within the temporal lobe. J Neurosci 24:5901-5908.

Wixted JT (2004) The psychology and neuroscience of forgetting. Annu Rev Psychol 55:235-269.

Young SL, Bohenek DL, Fanselow MS (1995) Scopolamine impairs acquisition and facilitates consolidation of fear conditioning: differential effects for tone vs context conditioning. Neurobiol Learn Mem 63:174-180.

Zola-Morgan S, Squire LR, Amaral DG, Suzuki WA (1989) Lesions of perirhinal and parahippocampal cortex that spare the amygdala and hippocampal formation produce severe memory impairment. J Neurosci 9:4355-4370. 